\title{
Role of Virtual Reality in Balance Training in Patients with Spinal Cord Injury: A Prospective Comparative Pre-Post Study
}

\author{
Shilpasree Saha \\ Department of Physiotherapy, Maharishi Markandeshwar Institute of Physiotherapy and Rehabilitation, Mullana, India
}

To the Editor,

I have read the article titled "Role of virtual reality in balance training in patients with spinal cord injury: a prospective comparative pre-post study," by Sengupta et al. [1]. The study discusses the use of virtual reality in balance training. However, I would like some of my concerns and queries to be addressed in this issue.

The sample size for this study was calculated based on a pilot study. The effect sizes for pre-post differences in the outcome measures from the Berg Balance Scale (BBS), Functional Reach Score (FRS), and balance section of the Tinetti Performance-Oriented Mobility Assessment (POMA-B) were found to be $0.81,1.25$, and 1.22 , respectively. Assuming an acceptable alpha error of 0.5 and aiming for $95 \%$ power of the study, the authors found the sample sizes for a two-tailed hypothesis was 22,11 , and 11 for the three outcomes, respectively. However, when the sample sizes for BBS, FRS, and POMA-B were 34, 14, and 16, respectively, when calculated using the $G^{\star}$ Power software (Heinrich-Heine-Universität Düsseldorf, Düsseldorf, Germany; http://www.gpower.hhu.de/) based on the above criteria [2].

In addition, on page 54, Fig. 1 of Sengupta et al. [1], the authors mentioned follow-up in all areas of allocation, follow-up, and analysis, which are not appropriate [3].

The authors did not test for normality because of small sample size. According to central limit theorem, violation of normality is not a major issue with sample size $\geq 100$, but to draw meaningful conclusions, assumption of normality should be followed regardless of the sample size. Continuous data with a normal distribution should be presented as mean values, and significance levels ( $p$-values) between and among the groups should be calculated using the mean value. However, for data with non-normal distribution, the resultant mean is not a representative of the data. Selecting the wrong representative data and calculating the significance level using this value might lead to a wrong interpretation. Therefore, testing for normality of data is important. The decision can be taken as to whether the mean is applicable as a representative value of the data. If applicable, a parametric test is used to compare means, otherwise medians are used to compare the groups using nonparametric methods [4].

\section{Conflict of Interest}

No potential conflict of interest relevant to this article was reported. 


\section{References}

1. Sengupta M, Gupta A, Khanna M, Rashmi Krishnan UK, Chakrabarti D. Role of virtual reality in balance training in patients with spinal cord injury: a prospective comparative pre-post study. Asian Spine J 2020;14:51-8.

2. Garg R, Malhotra V, Tripathi Y, Agarawal R. Effect of left, right and alternate nostril breathing on verbal and spatial memory. J Clin Diagn Res 2016;10:CC013.
3. Scazufca M, de Paula Couto MC, Henrique MG, et al. Pilot study of a two-arm non-randomized controlled cluster trial of a psychosocial intervention to improve late life depression in socioeconomically deprived areas of Sao Paulo, Brazil (PROACTIVE): feasibility study of a psychosocial intervention for late life depression in Sao Paulo. BMC Public Health 2019;19:1152.

4. Mishra P, Pandey CM, Singh U, Gupta A, Sahu C, Keshri A. Descriptive statistics and normality tests for statistical data. Ann Card Anaesth 2019;22:67-72. 